\title{
Perspective
}

Ashley R. Hoover, Kaili Liu and Wei R. Chen*

\section{Balance and modulation of immunoediting for cancer treatment using synergistic nano-photo-immuno effects}

https://doi.org/10.1515/nanoph-2021-0390

Received July 20, 2021; accepted August 13, 2021;

published online August 27, 2021

\begin{abstract}
Nanotechnology, photonics, and immunotherapy are far-reaching technologies with the potential to revolutionize the field of cancer diagnostics and therapeutics. While each technology has limitations in cancer treatment, they can be synergized to exert profound impact on the balance and modulation of immunoediting in tumor microenvironment (TME) and in the entire host immune system. We provide our perspectives on how nano-photoimmuno interactions can be used as an effective therapy, particularly when combined with other treatment modalities, such as checkpoint immune therapy, chemotherapy, and TME modulation, to provide a long-term, tumor-specific immunity against tumor metastasis and tumor recurrence.
\end{abstract}

Keywords: immunoediting; immunotherapy; metastatic cancers; nanotechnology; photonics; tumor microenvironment.

The concept of cancer immunotherapy is not new, but the idea of using specific immune cell types to combat cancer is a modern concept. William Coley, a surgeon in 19th century New York City, is credited with founding cancer immunotherapy. Deeply affected by the loss of a young cancer patient, Coley did a thorough investigation of case studies in cancer patients and discovered a link between bacterial infection and cancer regression. More than a hundred years ago, in a bold attempt to provide cancer patients with some form of treatment regimen, Dr. Coley developed a cocktail of Gram-negative and Gram-positive heat-killed bacteria known as the "Coley Toxin". He directly injected this

\footnotetext{
*Corresponding author: Wei R. Chen, Stephenson School of Biomedical Engineering, University of Oklahoma, Norman, OK 73019, USA, E-mail: Wei-R-Chen@ou.edu. https://orcid.org/0000-00027133-5794

Ashley R. Hoover and Kaili Liu, Stephenson School of Biomedical Engineering, University of Oklahoma, Norman, OK 73019, USA
}

mixture into the tumor to initiate a high fever over a period of several days and noticed that the solid tumors would become softer, change color, sometime ulcerate, and ultimately undergo necrosis, resulting in cancer regression. With this simple treatment, he had remarkable success, noting several cases in which some patients were cured while in others there was initial success on the treated tumors despite later recurrence in other locations [1, 2]. All of this was done without the knowledge that the immune system was being triggered to kill the tumor.

Despite Coley's initial, limited success in working towards a cancer vaccine, little progress was achieved following his death for several decades. This was largely attributed to the fact that the immune system remained largely unstudied until the late 1960s, when T cells were discovered by Jacques Miller [3], followed by the characterization of the antigen presenting cell, dendritic cells, by Ralph Steinman [4, 5]. Despite these seminal discoveries, little attention was given to the concept of cancer immunotherapy, likely owing to the belief that tumors are "self" tissues and are unlikely to stimulate an immune response, despite tumor cells undergoing random mutations. It was not until 1998 when Schreiber and colleagues presented concrete evidence of immune surveillance $[6,7]$ and expounded upon the immune surveillance hypothesis posited independently by Sir MacFarlane Burnet and Lewis Thomas in the 1950s [8] that cancer immunotherapy really became a topic worth investigating.

To date there are five different classes of cancer immunotherapies, many of which have been around for decades [9]. These include immunomodulators, antibody targeted cell elimination, vaccines, oncolytic viruses, and cell-based immunotherapies [9-12]. The first cell-based immunotherapy to treat hematologic cancers was performed at the University of Minnesota in 1970s in the form of bone marrow transplantation [13]. This was followed by the approval of the first immunomodulator, interferon alpha (IFN $\alpha$ ), for leukemia treatment in 1986 [14, 15]. Nearly 80 years after Coley's seminal work using bacteria to stimulate antitumor immunity, the bacillus Calmette- 
Guerin (BCG) tuberculosis vaccine was being used for bladder cancer treatment in the mid-1970s [15, 16]. In 1997, antibodies against the B cell surface marker CD20 were approved for lymphoma treatment, giving rise to antibodybased targeted therapeutics [17]. Lastly, in 2015 the oncolytic virus $\mathrm{T}$-Vec was approved to treat advanced lymphoma patients $[18,19]$.

Due to the success of these therapies in prolonging patient survival, cancer immunotherapy has recently become accepted as a main-stream therapy, particularly when the journal Science named cancer immunotherapy the "Breakthrough of the Year" in 2013 [20] and, subsequently, when the 2018 Nobel Prize in Physiology or Medicine was awarded to the pioneers of the "discovery of cancer therapy by inhibition of negative immune regulation" [21]. Specifically, leading to the epitome of cancer immunotherapy is the discovery of immune regulating ligands coined immune "checkpoints". To maintain immune homeostasis at steady-state or following an infection, the immune system has evolved ways to turn off or tune down the immune response through these immune checkpoints. There are many cell surface checkpoint molecules expressed on the surface of activated CD4 and CD8 $\mathrm{T}$ cells, but the most famous are cytotoxic $\mathrm{T}$ lymphocyte associated protein 4 (CTLA-4) and programmed cell death 1 (PD-1) [22, 23]. These molecules can be highly expressed on tumor infiltrating $\mathrm{T}$ cells, and actively prevent their tumoricidal functions. The major focus of immune checkpoint therapy (ICT) is to block these surface checkpoint proteins on immune cells to prevent their immunosuppressive signaling. Another major function of ICT is to block checkpoint proteins, such as programmed cell death ligand 1 (PD-L1), on tumor surfaces. PD-L1 binds to PD-1 to prevent T cell mediated tumor killing, in response to interferon gamma (IFNy) [23, 24]. Blocking PD-L1 interactions with PD-1 prevents the inhibitory signal from binding and tuning down the $\mathrm{T}$ cell response.

While the immunotherapies, particularly ICT, seek to eliminate tumors by releasing the brakes on $\mathrm{T}$ cells that specifically recognize tumor cells, they have failed to achieve satisfactory outcomes in clinical studies thus far $[25,26]$. To understand the current limitations of ICT we must fully understand the tumor microenvironment (TME) and its interplay with the entire immune system. Immune cells, tumor cells, the extracellular matrix (EM), TME metabolites, and cancer associated fibroblasts (CAFs) are intimately interconnected and can influence each other [27-30]. This concept is illustrated in Shreiber's hypothesis of "Cancer Immunoediting" which is an immune-centric hypothesis to describe the dual role of the immune system in inhibiting and promoting tumor progression [31]. Cancer immunoediting is divided into three sequential phases known as the three E's: elimination, equilibrium, and escape [32, 33]. During the elimination phase, which expounds upon the initial immune surveillance hypothesis, the innate and adaptive immune systems work in concert to detect and destroy tumors, preventing uncontrolled tumor growth. The equilibrium phase suggests that the adaptive immune system, which is capable of recognizing specific tumor antigens, inadvertently selects tumor cells capable of surviving the initial immune attack/recognition. In this phase the immune cells co-exist with the tumor cells, which remain dormant, creating an equilibrium between the two camps. During the escape phase, these dormant tumor cells become active either by actively preventing immune recognition/destruction, or by establishing an immunosuppressive TME that actively prevents tumor killing and enhances tumor growth [32-34].

The hypothesis of cancer immunoediting is nicely illustrated in human cancer responses to cancer treatment, particularly ICT. Patients receiving ICT that do not achieve long-term benefit can be divided into three categories: primary resistance, adaptive resistance, and acquired resistance [35-37]. The primary resisters do not respond to immunotherapy due to both tumor intrinsic and tumor extrinsic factors in TME. The adaptive resisters have tumor immune recognition, but the tumor actively adapts to circumvent immune attack, effectively creating an arms race between the immune system and the TME. The acquired resisters initially respond well to ICT, but then relapse after a period of time despite continuous therapy. All three types of resistance to ICT have a close connection with TME.

Characterizing the evolvement of the TME following ICT and other immunotherapies, as well as common tumor resistance mechanisms can guide us in the development of combinational therapies to overcome therapeutic resistance. The TME has multiple complementing immunosuppressive components. If one component is removed, the remaining components compensate for that loss. These compensatory mechanisms limit the success of celltargeted therapy in solid tumors. Therefore, the key is to design a "smart" targeted therapy that can tackle the compensatory mechanisms that exist within the TME. One promising approach is an ablative therapy combining photonics, nanotechnology and immunotherapy, adaptable specifically to the targeted tumor.

Photonics and nanotechnology were arguably two of the most far-reaching scientific and technological 
advances in the 20th century. Lasers have opened new doors in diagnostics and therapeutics. Particularly in cancer treatment, photothermal therapy (PTT) and photodynamic therapy (PDT) have become major tools with impressive outcomes [38-42]. In addition to the direct tumor cell destruction via photothermal interactions through PTT and photochemical interactions through PDT, phototherapy also induces immunogenic cell death, leading to the release of tumor associated antigens (TAAs), tumor specific antigens (TSAs), and danger associated molecular patterns (DAMPs) [43, 44]. Additionally, targeted ablation has another important function: it can disrupt the established TME, potentiating immune cell infiltration and tumor killing. However, the impacts on the TME by phototherapies alone have achieved limited success in treating cancers, particularly metastatic cancers.

By using phototherapy alone for cancer treatment, we tend to lose sight that the tumor is self-tissue, to which the adaptive immune system is selected not to respond. While tumor antigens are immunogenic, because TSAs can generate $\mathrm{T}$ cell responses capable of eliminating tumors, we hypothesize that these immunogenic TSAs are far less abundant than "self" antigens. To generate an effective systemic response to the TSAs, addition of a strong immune stimulant is required. This is evidenced by the additive, often synergistic, effect observed when PTT or PDT is combined with an immunostimulant, resulting in enhanced elimination of treated primary tumors and untreated metastases [45-47]. However, in many cases, this combination can enhance animal survival but not eliminate the targeted tumor [48-50]. While the reasons for this incomplete elimination of the tumors have not been widely explored in detail, the tumor intrinsic and extrinsic factors that contribute to resistance to immunotherapy treatment in humans likely play a dominant role.

Nanoparticles have been used for years in PTT and PDT $[48,51,52]$. They have been used as light-absorbers for PTT, photosensitizers for PDT, and/or as carriers of phototherapy agents specifically to target tumor cells. Recently, however, more investigators have been taking advantage of the dynamic nature of nanoparticles and using them as a multipurpose tool to deliver TME modulators, chemotherapeutic drugs, and/or immune stimulants simultaneously and specifically to tumors $[53,54]$. This delivery system reduces off-target effects, improves pharmacokinetics, and limits side effects imposed by systemic treatments. While nanoparticles loaded with tumoricidal cargos have achieved certain success, they have not resulted in high curative potential when not combined with ablation [55-57].

Nanotechnology-based ablative immunotherapies can be a targeted, multifaceted approach to overcome such limitations. Immune-ablation combined with nanoparticles can help overcome tumor intrinsic and extrinsic factors. We term this combinatorial approach nano-ablative immunotherapy (NAIT). The hypothesis behind NAIT is that the nanoparticles augment phototherapy for tumor ablation to disrupt TME homeostasis, releasing TAAs, TSAs, DAMPs, and stimulating recruitment of new immune cells to the TME. Adding the immune stimulation following ablation enhances the immune response to TSAs and ensures that the recruited immune cells in the treated tumor polarize into an inflammatory phenotype. Therefore, nanoparticles ensure the specificity of the tumor ablation and the specific release of the immune stimulants. In addition, the nanoparticles should also release cargos that directly target the tumor cells and/or the TME to prevent the remaining tumor cells from adapting and reestablishing an immunosuppressive TME.

Specifically, the locally administered nanoparticles, TME modulators, chemotherapeutics, and/or immunostimulants, either before and/or after photoablation, could effectively remodel the immunosuppressive TME, hence successfully modulating the co-evolvement between tumor cells and immune cells in the TME. The concept of NAIT is depicted in Figure 1. As shown in Figure 1, the optimal approach is to start with delivery of a nanoplatform carrying different therapeutic agents, particularly immunological stimulants, chemotherapeutics, and TME modulators, to the target tumor. The phototherapy-potentiated tumor ablation will destroy tumors and induce immunogenic tumor cell death to recruit immune cells. Any residual tumor/stromal cells left over from ablation will then be targeted by the chemotherapeutics, while the TME modulators help prevent the reestablishment of an immunosuppressive TME. The nanoplatform-carried immunostimulant, when combined with the released tumor antigens and DAMPs, amplifies the proinflammatory activation of immune cells and antigen-presenting cells (APCs), such as dendritic cells (DCs). The matured APCs can then present the tumor antigens to the T cells in tumor-draining lymph nodes (TDLNs) for T cell activation and proliferation. Finally, these $\mathrm{T}$ cells, primed against the treated tumors, can enter the TME and eliminate the residual tumors at the treatment site and the untreated distal metastases. A similar process can also occur in the spleen, which is where $\mathrm{T}$ cells also develop and are critical for the effectiveness of immunotherapies $[58,59]$. 


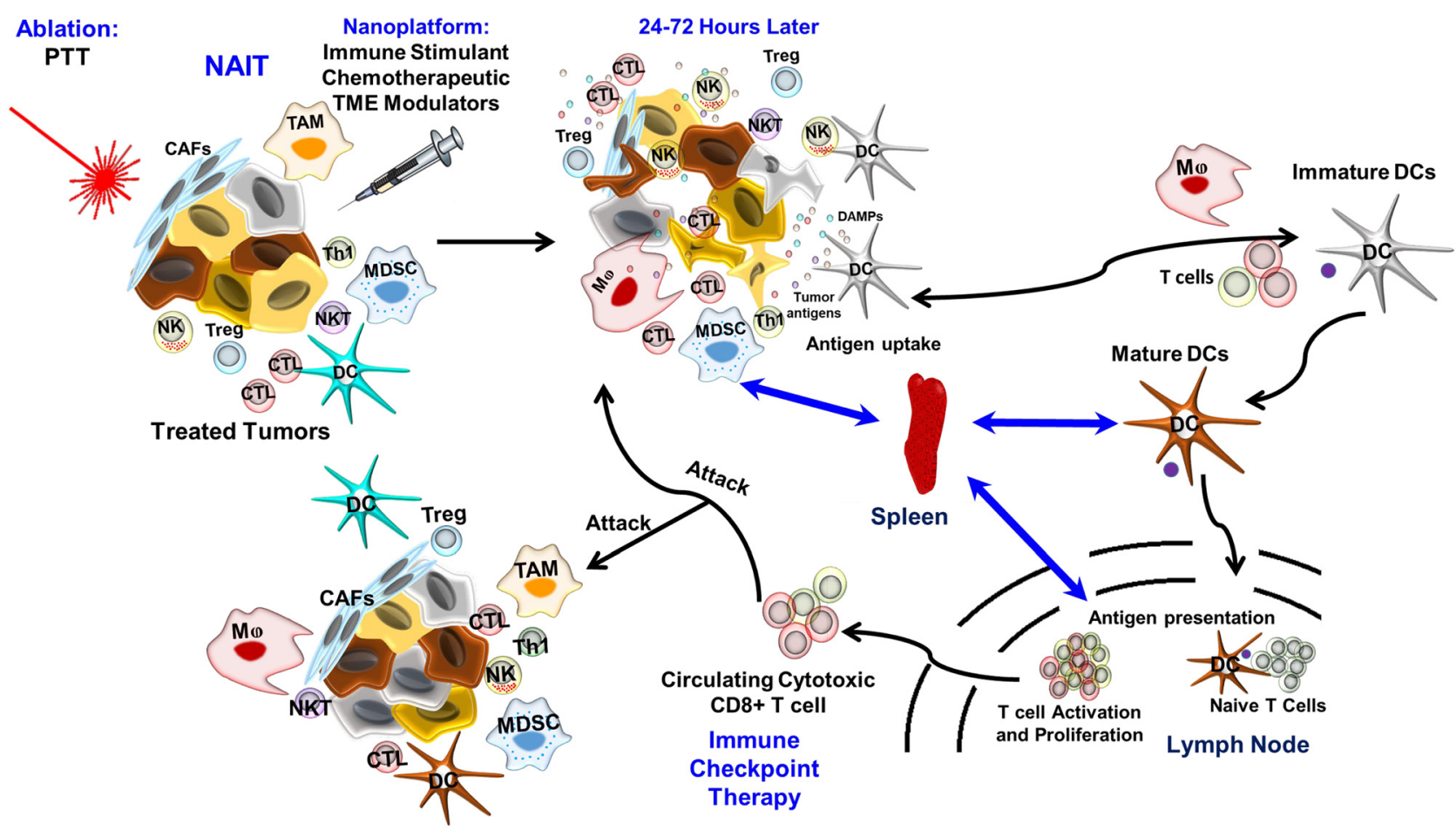

Untreated Metastases

Figure 1: Illustration of nano-ablative-immunotherapy (NAIT) for the treatment of primary and metastatic tumors, through spatiotemporally coordinated immune responses and immune checkpoint therapy.

Photothermal therapy (PTT) disrupts tumor homeostasis, initiates immunogenic cell deaths (ICDs) that release danger associated molecular patterns (DAMPs) and tumor antigens, and recruit immune cells to the tumor. The addition of immune stimulants ensures that the immune cells recruited to the tumor polarize into an antitumor response. The adaptive antitumor responses can be further enhanced by the addition of immune checkpoint therapy (ICT). The addition of chemotherapeutics will target the remaining tumor cells and the untreated metastases for ICD resulting in the recruitment of inflammatory cells to the site of death. In the treated tumors, tumor microenvironment (TME) modulators will help prevent the reestablishment of an immunosuppressive TME. In the metastatic tumors, they can suppress the immunosuppressive TME, allowing for more efficient tumor killing of the newly recruited antitumor immune cells which can be further enhanced via the immune stimulant. CTL, cytotoxic lymphocyte; TAM, tumor associated macrophages; Treg, T regulatory cell; DC, dendritic cell; NK, natural killer cell; CAF, cancer associated fibroblast; NKT, natural killer T cell; Mœ, inflammatory macrophage; Th1, T helper $1 \mathrm{~T}$ cell.

In scenario depicted in Figure 1, the TME modulators and the chemotherapeutics play a much more significant role in the untreated metastases, allowing for recruitment of inflammatory cells into the TME through chemotherapy induced ICD of the tumor cells. Furthermore, the TME modulators will help suppress the immunosuppressive functions of metabolites and cytokines within the TME, enhancing the antitumor function of the recruited antitumor immune cells. The immune stimulant will further recruit inflammatory immune cells to enter the metastatic TME and help prolong/enhance their tumor killing activity to eventually eliminate the tumor or keep it from growing. One important fact is that each tumor is unique, and the TME is ever evolving and adapting to prevent immune mediated killing. Additionally, TMEs of the metastases can be different from TMEs of the primary tumors. The nanoparticles used in NAIT can potentially overcome these issues as they are customizable and can be combined with a variety of chemotherapeutics, TME modulators, antiinflammatory cytokines and metabolite inhibitors, and/or immune modulators to enhance the elimination of the untreated metastases, as shown in Figure 2.

In summary, the combination of nanotechnology, photonics, and immunotherapy possesses great potential in cancer treatment. It can balance the 3 Es of the immunoediting process by positively shaping the TME and the entire immune system. It also primes the immune system for other therapies, particularly ICT. The future studies 

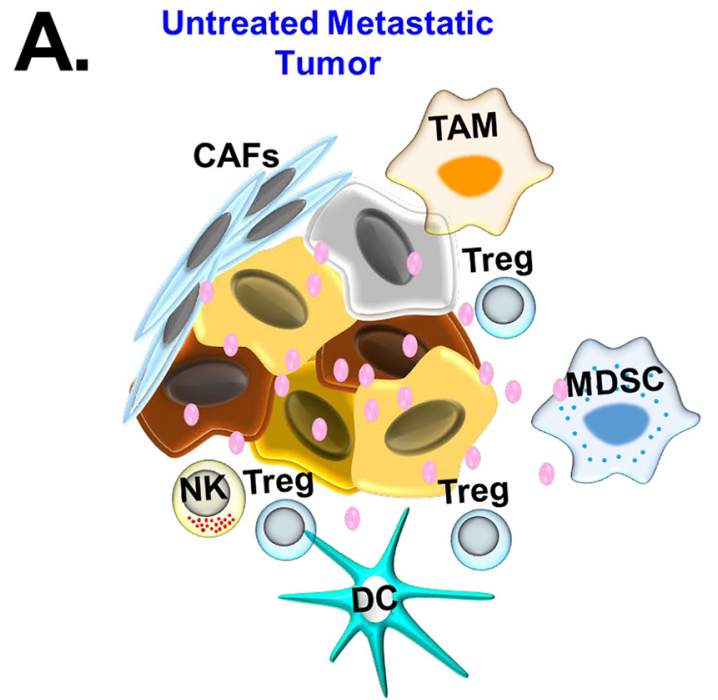

Nanoparticles

Loaded with

chemotherapeutics,

immune stimulants,

and TME modulators

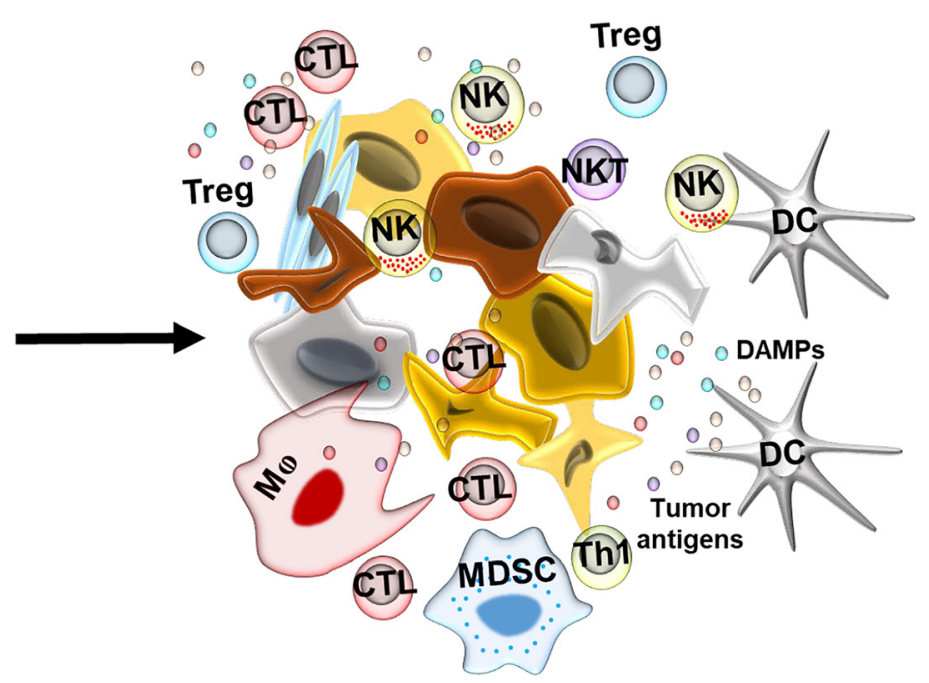

Enhanced Tumor killing

B.

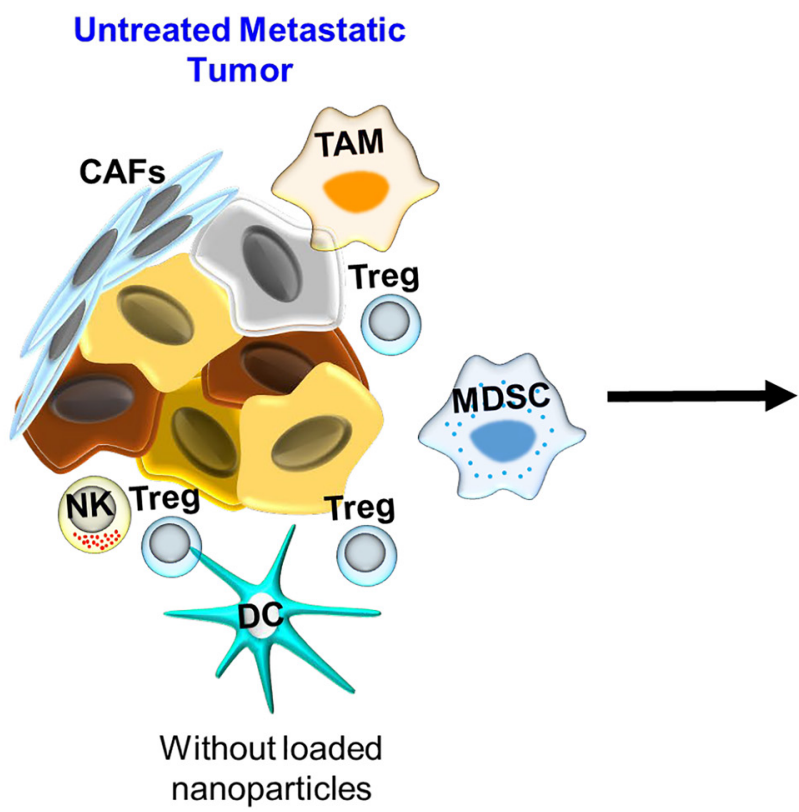

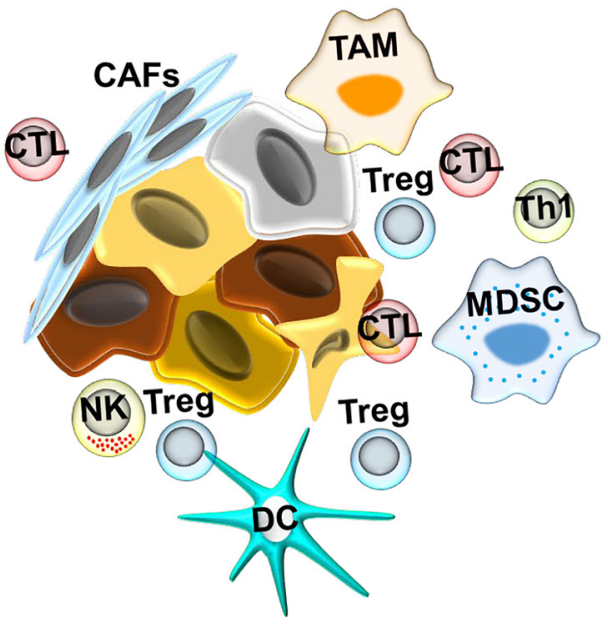

Minimal Tumor killing

Figure 2: Illustration of the addition of nanoparticles loaded with chemotherapeutics, immune stimulants, and TME modulators, as well as their effects on the untreated metastatic tumors.

A. The chemotherapeutics result in immunogenic cell death and the release of tumor antigens and DAMPs, while the immune stimulants enhance the antitumor immune responses generated by the tumor antigens and DAMPs. The TME modulators help sustain tumor killing and prevent and/or slow the reestablishment of the immunosuppressive TME. B. Without the addition of nanoparticles loaded with the therapeutic cargos, the immune response to the metastases is limited since the TME is not altered apart from a few CTLs. CTL, cytotoxic lymphocyte; TAM, tumor associated macrophages; Treg, T regulatory cell; DC, dendritic cell; NK, natural killer cell; CAF, cancer associated fibroblast; NKT, natural killer T cell. 
should aim at taking full advantage of developing optimal combinatorial approaches using these technologies for clinical applications to treat cancer.

Author contributions: All the authors have accepted responsibility for the entire content of this submitted manuscript and approved submission.

Research funding: This work was supported in part by the U.S. National Institutes of Health (R01CA205348) and the Oklahoma Center for the Advancement of Science and Technology (HR16-085 and HF20-019).

Conflict of interest statement: The authors declare no conflicts of interest regarding this article.

\section{References}

[1] W. B. Coley, "II. Contribution to the knowledge of sarcoma," Ann. Surg., vol. 14, no. 3, pp. 199-220, 1891.

[2] W. B. Coley, "The treatment of malignant tumors by repeated inoculations of erysipelas. With a report of ten original cases. 1893," Clin. Orthop. Relat. Res., vol. 262, pp. 3-11, 1991.

[3] W. K. Decker, R. F. da Silva, M. H. Sanabria, et al., "Cancer immunotherapy: historical perspective of a clinical revolution and emerging preclinical animal models," Front. Immunol., vol. 8, no. 829, 2017, https://doi.org/10.3389/fimmu.2017.00829.

[4] J. F. A. P. Miller, G. F. Mitchell, and N. S. Weiss, "Cellular basis of the immunological defects in thymectomized mice," Nature, vol. 214, no. 5092, pp. 992-997, 1967.

[5] R. M. Steinman and Z. A. Cohn, "Identification of a novel cell type in peripheral lymphoid organs of mice. I. Morphology, quantitation, tissue distribution," J. Exp. Med., vol. 137, no. 5, pp. 1142-1162, 1973.

[6] R. M. Steinman and Z. A. Cohn, "Identification of a novel cell type in peripheral lymphoid organs of mice. II. Functional properties in vitro," J. Exp. Med., vol. 139, no. 2, pp. 380-397, 1974.

[7] D. H. Kaplan, V. Shankaran, A. S. Dighe, et al., "Demonstration of an interferon $\gamma$-dependent tumor surveillance system in immunocompetent mice," Proc. Natl. Acad. Sci. U. S. A., vol. 95, no. 13, pp. 7556-7561, 1998.

[8] V. Shankaran, H. Ikeda, A. T. Bruce, et al., "IFNY and lymphocytes prevent primary tumour development and shape tumour immunogenicity," Nature, vol. 410, no. 6832, pp. 1107-1111, 2001.

[9] D. Ribatti, "The concept of immune surveillance against tumors. The first theories," Oncotarget, vol. 8, no. 4, pp. 7175-7180, 2017.

[10] L. Galluzzi, E. Vacchelli, J-M. Bravo-San Pedro, et al., "Classification of current anticancer immunotherapies," Oncotarget, vol. 5, no. 24, pp. 12472-12508, 2014.

[11] N. Margolis, E. Markovits, and G. Markel, “Reprogramming lymphocytes for the treatment of melanoma: from biology to therapy," Adv. Drug Deliv. Rev., vol. 141, pp. 104-124, 2019.

[12] J. Martin-Liberal, M. Ochoa de Olza, C. Hierro, A. Gros, J. Rodon, and J. Tabernero, "The expanding role of immunotherapy," Canc. Treat Rev., vol. 54, pp. 74-86, 2017.

[13] M. Khan, A. V. Maker, and S. Jain, "The evolution of cancer immunotherapy," Vaccines, vol. 9, no. 6, p. 614, 2021.
[14] L. A. Kujawski and M. Talpaz, "The role of interferon-alpha in the treatment of chronic myeloid leukemia," Cytokine Growth Factor Rev., vol. 18, no. 5, pp. 459-471, 2007.

[15] M. Talpaz, R. Hehlmann, A. Quintás-Cardama, J. Mercer, and J. Cortes, "Re-emergence of interferon- $\alpha$ in the treatment of chronic myeloid leukemia," Leukemia, vol. 27, no. 4, pp. 803-812, 2013.

[16] H. W. Herr and B. C. G. Intravesical, "Current results, natural history and implicants for urothelial cancer prevention," J. Cell. Biochem., vol. 50, no. S16I, pp. 112-119, 1992.

[17] C. Luo, G. Wu, X. Huang, et al., "Efficacy and safety of new anti-CD20 monoclonal antibodies versus rituximab for induction therapy of CD20+ B-cell non-Hodgkin lymphomas: A systematic review and meta-analysis," Sci. Rep., vol. 11, no. 1, p. 3255, 2021.

[18] P. Feugier, "A review of rituximab, the first anti-CD20 monoclonal antibody used in the treatment of B non-Hodgkin's lymphomas," Future Oncol., vol. 11, no. 9, pp. 1327-1342, 2015.

[19] H. Rehman, A. W. Silk, M. P. Kane, and H. L. Kaufman, "Into the clinic: talimogene laherparepvec (T-VEC), a first-in-class intratumoral oncolytic viral therapy," J. Immunother. Canc., vol. 4, no. 1, p. 53, 2016.

[20] J. Couzin-Frankel, "Cancer immunotherapy," Science, vol. 342, no. 6165, pp. 1432-1433, 2013.

[21] Z. S. Guo, "The 2018 Nobel Prize in medicine goes to cancer immunotherapy (editorial for BMC cancer)," BMC Canc., vol. 18, no. 1, p. 1086, 2018.

[22] A. C. Anderson, N. Joller, and V. K. Kuchroo, "Lag-3, tim-3, and TIGIT: Co-inhibitory receptors with specialized functions in immune regulation," Immunity, vol. 44, no. 5, pp. 989-1004, 2016.

[23] A. D. Waldman, J. M. Fritz, and M. J. Lenardo, "A guide to cancer immunotherapy: from T cell basic science to clinical practice," Nat. Rev. Immunol., vol. 20, no. 11, pp. 651-668, 2020.

[24] Y. Iwai, M. Ishida, Y. Tanaka, T. Okazaki, T. Honjo, and N. Minato, "Involvement of PD-L1 on tumor cells in the escape from host immune system and tumor immunotherapy by PD-L1 blockade," Proc. Natl. Acad. Sci. U. S. A., vol. 99, no. 19, pp. 12293-12297, 2002.

[25] A. Haslam and V. Prasad, "Estimation of the percentage of US patients with cancer who are eligible for and respond to checkpoint inhibitor immunotherapy drugs," JAMA Netw. Open, vol. 2, no. 5, p. e192535, 2019.

[26] Y. Zou, X. Zou, S. Zheng, et al., "Efficacy and predictive factors of immune checkpoint inhibitors in metastatic breast cancer: $\mathrm{A}$ systematic review and meta-analysis," Ther. Adv. Med. Oncol., vol. 12, 2020, Art no. 1758835920940928.

[27] S. L. Topalian, F. S. Hodi, J. R. Brahmer, et al., "Safety, activity, and immune correlates of anti-PD-1 antibody in cancer," N. Engl. J. Med., vol. 366, no. 26, pp. 2443-2454, 2012.

[28] M. Binnewies, E. W. Roberts, K. Kersten, et al., “Understanding the tumor immune microenvironment (TIME) for effective therapy," Nat. Med., vol. 24, no. 5, pp. 541-550, 2018.

[29] D. Hanahan and M. Coussens Lisa, "Accessories to the crime: functions of cells recruited to the tumor microenvironment," Canc. Cell, vol. 21, no. 3, pp. 309-322, 2012.

[30] S. J. Turley, V. Cremasco, and J. L. Astarita, "Immunological hallmarks of stromal cells in the tumour microenvironment," Nat. Rev. Immunol., vol. 15, no. 11, pp. 669-682, 2015. 
[31] R. D. Schreiber, L. J. Old, and M. J. Smyth, "Cancer immunoediting: integrating immunity's roles in cancer suppression and promotion," Science, vol. 331, no. 6024, pp. 1565-1570, 2011.

[32] G. P. Dunn, L. J. Old, and R. D. Schreiber, "The three Es of cancer immunoediting," Annu. Rev. Immunol., vol. 22, pp. 329-360, 2004.

[33] D. Mittal, M. M. Gubin, R. D. Schreiber, and M. J. Smyth, “New insights into cancer immunoediting and its three component phases-elimination, equilibrium and escape," Curr. Opin. Immunol., vol. 27, pp. 16-25, 2014.

[34] J. S. O’Donnell, M. W. L. Teng, and M. J. Smyth, “Cancer immunoediting and resistance to T cell-based immunotherapy," Nat. Rev. Clin. Oncol., vol. 16, no. 3, pp. 151-167, 2019.

[35] P. Sharma, S. Hu-Lieskovan, J. A. Wargo, and A. Ribas, "Primary, adaptive, and acquired resistance to cancer immunotherapy," Cell, vol. 168, no. 4, pp. 707-723, 2017.

[36] J. Veldman, L. Visser, Avd. Berg, and A. Diepstra, "Primary and acquired resistance mechanisms to immune checkpoint inhibition in Hodgkin lymphoma," Canc. Treat Rev., vol. 82, p. 101931, 2020.

[37] T. Oba, M. D. Long, T. Keler, et al., "Overcoming primary and acquired resistance to anti-PD-L1 therapy by induction and activation of tumor-residing cDC1s," Nat. Commun., vol. 11, no. 1, p. $5415,2020$.

[38] S. M. Schieke, P. Schroeder, and J. Krutmann, “Cutaneous effects of infrared radiation: from clinical observations to molecular response mechanisms," Photodermatol. Photoimmunol. Photomed., vol. 19, no. 5, pp. 228-234, 2003.

[39] D. van Straten, V. Mashayekhi, H. S. de Bruijn, S. Oliveira, and D. J. Robinson, "Oncologic photodynamic therapy: basic principles, current clinical status and future directions," Cancers, vol. 9, no. 2, p. 19, 2017.

[40] H. O. Alsaab, M. S. Alghamdi, A. S. Alotaibi, et al., "Progress in clinical trials of photodynamic therapy for solid tumors and the role of nanomedicine," Cancers, vol. 12, no. 10, p. 2793, 2020.

[41] M. F. Naylor, F. Zhou, B. V. Geister, R. E. Nordquist, X. Li, and W. R. Chen, "Treatment of advanced melanoma with laser immunotherapy and ipilimumab," J. Biophot., vol. 10, no. 5, pp. 618-622, 2017.

[42] M. F. Naylor, W. R. Chen, T. K. Teague, L. A. Perry, and R. E. Nordquist, "In situ photoimmunotherapy: A tumourdirected treatment for melanoma," Br. J. Dermatol., vol. 155, no. 6, pp. 1287-1292, 2006.

[43] W. Li, J. Yang, L. Luo, et al., "Targeting photodynamic and photothermal therapy to the endoplasmic reticulum enhances immunogenic cancer cell death," Nat. Commun., vol. 10, no. 1, p. 3349, 2019.

[44] J. R. Melamed, R. S. Edelstein, and E. S. Day, "Elucidating the fundamental mechanisms of cell death triggered by photothermal therapy," ACS Nano, vol. 9, no. 1, pp. 6-11, 2015.

[45] Y. Zhang, X. Zhan, J. Xiong, et al., "Temperature-dependent cell death patterns induced by functionalized gold nanoparticle photothermal therapy in melanoma cells," Sci. Rep., vol. 8, no. 1, p. 8720, 2018.

[46] M. Korbelik, T. Hode, S. S. K. Lam, and W. R. Chen, "Novel immune stimulant amplifies direct tumoricidal effect of cancer ablation therapies and their systemic antitumor immune efficacy," Cells, vol. 10, no. 3, p. 492, 2021.

[47] Y. Li, X. Li, F. Zhou, et al., "Nanotechnology-based photoimmunological therapies for cancer," Canc. Lett., vol. 442, pp. 429-438, 2019.

[48] Q. Chen, L. Xu, C. Liang, C. Wang, R. Peng, and Z. Liu, "Photothermal therapy with immune-adjuvant nanoparticles together with checkpoint blockade for effective cancer immunotherapy," Nat. Commun., vol. 7, no. 1, p. 13193, 2016.

[49] B. Zhou, Q. Wu, M. Wang, et al., "Immunologically modified $\mathrm{MnFe}(2) \mathrm{O}(4)$ nanoparticles to synergize photothermal therapy and immunotherapy for cancer treatment," Chem. Eng. J., vol. 396, 2020, https://doi.org/10.1016/j.cej.2020.125239.

[50] X. Huang, Y. Lu, M. Guo, S. Du, and N. Han, “Recent strategies for nano-based PTT combined with immunotherapy: from a biomaterial point of view," Theranostics, vol. 11, no. 15, pp. 7546-7569, 2021.

[51] Y. Cheng, A. C. S., J. D. Meyers, I. Panagopoulos, B. Fei, and C. Burda, "Highly efficient drug delivery with gold nanoparticle vectors for in vivo photodynamic therapy of cancer,"J. Am. Chem. Soc., vol. 130, no. 32, pp. 10643-10647, 2008.

[52] D. P. O’Neal, L. R. Hirsch, N. J. Halas, J. D. Payne, and J. L. West, "Photo-thermal tumor ablation in mice using near infraredabsorbing nanoparticles," Canc. Lett., vol. 209, no. 2, pp. 171-176, 2004.

[53] L. Bai, W. Yi, T. Sun, et al., "Surface modification engineering of two-dimensional titanium carbide for efficient synergistic multitherapy of breast cancer," J. Mater. Chem. B, vol. 8, no. 30, pp. 6402-6417, 2020.

[54] Q. Chen, J. Chen, Z. Yang, et al., "Nanoparticle-enhanced radiotherapy to trigger robust cancer immunotherapy," Adv. Mater., vol. 31, no. 10, p. 1802228, 2019.

[55] F. Zhou, M. Wang, T. Luo, J. Qu, and W. R. Chen, "Photo-activated chemo-immunotherapy for metastatic cancer using a synergistic graphene nanosystem," Biomaterials, vol. 265, p. 120421, 2021.

[56] D. Rosenblum, N. Joshi, W. Tao, J. M. Karp, and D. Peer, "Progress and challenges towards targeted delivery of cancer therapeutics," Nat. Commun., vol. 9, no. 1, p. 1410, 2018.

[57] R. van der Meel, E. Sulheim, Y. Shi, F. Kiessling, W. J. M. Mulder, and T. Lammers, "Smart cancer nanomedicine," Nat. Nanotechnol., vol. 14, no. 11, pp. 1007-1017, 2019.

[58] W. Mu, Q. Chu, Y. Liu, and N. Zhang, "A review on nano-based drug delivery system for cancer chemoimmunotherapy," NanoMicro Lett., vol. 12, no. 1, p. 142, 2020.

[59] M. H. Spitzer, Y. Carmi, N. E. Reticker-Flynn, et al., "Systemic immunity is required for effective cancer immunotherapy," Cell, vol. 168, no. 3, pp. 487-502.e415, 2017. 